\title{
The effects on peripheral nerve damage of the application of local and systemic erythropoietin
}

\author{
Sarica $\mathrm{S}^{1}$, Altun $\mathrm{I}^{2}$ \\ Department of Otorhinolaryngology, Sutcu Imam University Faculty of Medicine, Kahramanmaras, Turkey. \\ idrisaltun46@hotmail.com
}

\begin{abstract}
OBJECTIVE: To investigate the histopathological and biochemical effects of topical and systemic administrations of erythropoietin (EPO) on crush and transection type peripheral nerve injuries in an experimental rat model. METHODS: 128 male Wistar-Albino rats were allocated to 8 groups according to the route of administration of EPO (local/systemic) and type of peripheral nerve injury (crush/transection). groups were compared with respect to histopathological examination and number of axons as well as levels of IL-1 $\beta$, IL-6, and IL-10.

RESULTS: Groups receiving EPO either locally or intraperitoneally revealed less scar tissue formation index, lower number of inflammatory cells, reduced number of perineural fibroblasts and increased number of axons $(p<0.001$ for all). Levels of IL-1 $\beta$ and IL-6 were lower and IL-10 levels were higher in groups receiving EPO locally or intraperitoneally $(p<0.001)$.

CONCLUSION: latrogenic nerve injury has remained an area with few therapeutic options. Our results indicated that local and systemic applications of EPO might have a promising potential therapeutic agent for crush or transection type of peripheral nerve injuries. Dose, route of administration and indications should be elucidated in further prospective, randomized, controlled trials (Tab. 3, Fig. 4, Ref. 32). Text in PDF www.elis.sk. KEY WORDS: peripheral nerve, injury, crush, transection, erythropoietin, IL-1 $\beta$, IL-6, IL-10.
\end{abstract}

\section{Introduction}

The administration of erythropoietin (EPO) results in angiogenesis, myogenesis, increased oxidative enzyme activity and anti-apoptotic and anti-inflammatory reactions (1-7).Apart from in the red cell surface, erythropoietin receptor (Epo-R) is also expressed in a large variety of normal tissues. Erythropoietin, as well as its receptor, is present in the central and peripheral nervous system $(8,9)$. Epo- $R$ is found in certain axons and neuron bodies of the dorsal root ganglion, in endothelial cells, and in Schwann cells of the normal peripheral nerve. Erythropoietin is produced in the bodies and the axons of normal ganglions in the rat dorsal root and increased erythropoietin levels are seen in Schwann cells after peripheral nerve injury $(10,11)$. Erythropoietin has a direct and indirect effect on nerve cells, enhances antioxidative enzyme production, antagonizes glutamate cytotoxic action, metabolizes free radicals, normalizes cerebral blood flow, affects neurotransmitter release and stimulates neoangiogenesis (12).

There are very few previous studies on the application of systemic EPO on peripheral nerve system damage and repair models. To the best of our knowledge, there has been no previous study

\footnotetext{
${ }^{1}$ Department of Otorhinolaryngology, Sutcu Imam University Faculty of Medicine, Kahramanmaras, Turkey, and ${ }^{2}$ Department of Neurosurgery, Sutcu Imam University Faculty of Medicine, Kahramanmaras, Turkey

Address for correspondence: I. Altun, MD, Department of Neurosurgery, Kahramanmaras Sutcu Imam University Medical Faculty, Kahramanmaras, Turkey.

Phone +903442803398, Fax: +903442803409
}

which has evaluated local EPO application in crush injury and incision type injury in the peripheral nerve system, which has applied long-term systemic and local EPO together, which has evaluated the significant problems in nerve damage of perineural adhesions, scar development and inflammation, evaluated the histomorphological alignment of axon fibres in the injury site, or defined the relationship between the levels of pro-inflammatory cytokines IL-1 $\beta$, IL- 6 and the anti-inflammatory cytokine IL-10 with EPO treatment.

\section{Materials and methods}

\section{Experimental design}

This experimental trial was performed after the approval of the local Institutional Animal Care and Use Committee (17.04.2014/55). Maximum effort was spent to minimize the suffering of animals and to reduce the number of animals included. Animals were maintained at constant temperature $\left(20-22^{\circ} \mathrm{C}\right)$ and humidity (50-60\%) with a diurnal cycle of 12-hour light and dark periods. Ad libitum access to food and water was allowed. Strict adherence to the guidelines of the National Institute of Health for the care and use of laboratory animals was provided for all procedures.

A total of 128 male Wistar-Albino rats (weighing 200 to $250 \mathrm{~g}$ ) were allocated in 8 groups with respect to the procedure applied. Each group involved sixteen rats. Group I underwent crush injury of the right sciatic nerve with local application of isotonic saline; Group II received local application of EPO after crush injury; Group III underwent intraperitoneal administration of isotonic 


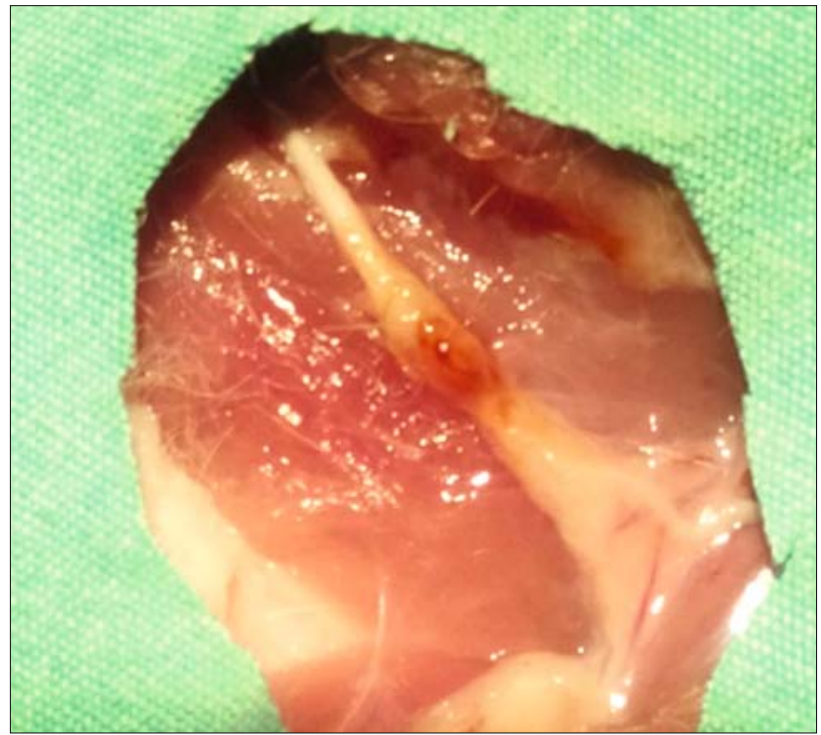

Fig. 1. The appearance of the sciatic nerve after formation of crush injury.

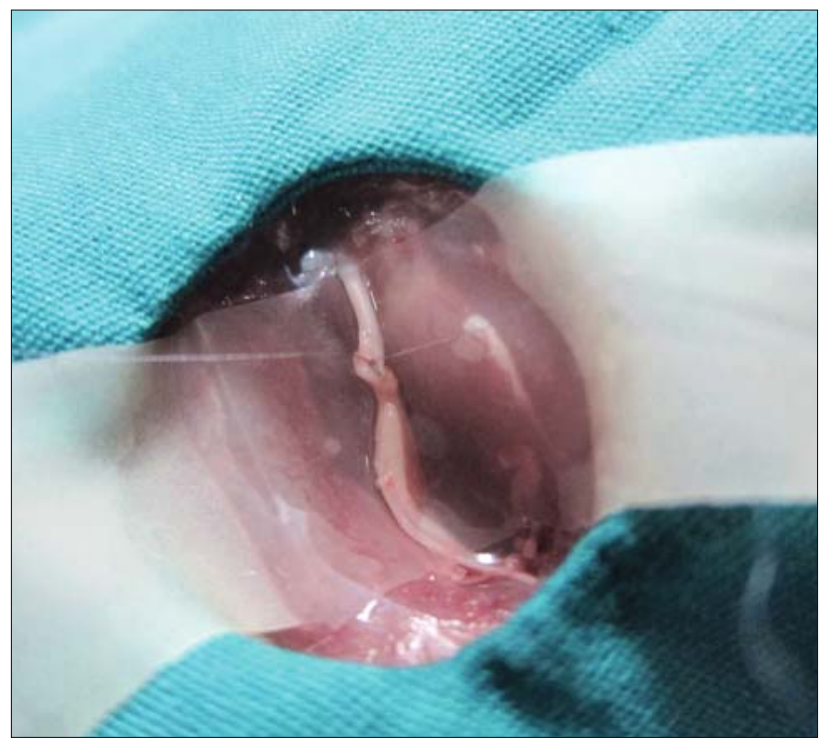

Fig. 2. End-to-end anastomosis of sciatic nerve with sutures after transection type injury.

saline after crush injury; Group IV had intraperitoneal EPO after crush injury; Group V received isotonic saline at the site of injury after transection of the sciatic nerve; Group VI underwent local application of EPO after transection; Group VII had intraperitoneal isotonic saline following transection and Group VIII received intraperitoneal EPO after transection type nerve injury.

Rats were anesthesized using an intraperitoneal injection of $15 \mathrm{mg} / \mathrm{kg}$ of xylazine and $100 \mathrm{mg} / \mathrm{kg}$ of ketamine, respectively (Bayer AG, Leverkusen, Germany). After a 12-week follow-up period, biopsies were obtained from the site of neural injury and groups were compared with respect to histopathological scoring based on inflammatory, degenerative and fibrotic changes. As de- scribed in the literature, intraperitoneal and topical injections of EPO were performed at a dose of $5000 \mathrm{U} / \mathrm{kg}$. On the other hand, control received $1 \mathrm{ml}$ of isotonic saline injections either at the site of injury or intraperitoneally (13).

All surgical interventions were carried out by the same surgeon (IA) with the same equipment under anesthesia. The right sciatic nerve was recognized at the mid-level of the right thigh. Transection type injury was created with full-thickness incision of sciatic nerve with a no. 15 scalpel was performed at the location $1 \mathrm{~cm}$ from the sciatic notch. Crush injury of the sciatic nerve was induced by the instrumented clamp ( $1 \mathrm{X}$ with $25 \mathrm{~N}$ for 30 seconds) as described by Yu et al (Fig. 1) (14). After formation of transection type injury on the sciatic nerve, the end-to-end anastomosis was made (Fig. 2). Following the reapposition of the muscular and cutaneous layers in anatomical planes, the wound was closed with fine sutures.

\section{Biochemical evaluation}

After a follow-up period of 6 weeks, eight animals from each sub-group were euthanized with an anesthetic overdose. The left sciatic nerves were removed from all the animals and processed for biochemical analysis. The left sciatic nerves of the control group animals were taken as samples of normal nerve tissue. IL-1 $\beta$, IL-6, and IL-10 with the ELISA (enzyme-linked immunosorbent assay) method using commercial kits (eBioscience Company, Vienna, Austria), an automatic ELISA microplate reader (ThermoScientific, Finland) and a computer program (Skanlt for Multiscan FC2.5.1). The results were expressed in picogram per gram tissue.

\section{Macroscopic evaluation}

At the end of a 12-week follow-up period, all the rats were re-anesthetized, and the surgical site of the groups was examined in detail by microdissection. Skin and muscle fascia adhesions and perineural adhesions were assessed by a researcher blinded to the groups and according to the numerical grading scheme as described by Peterson et al (15). Numerical grading system for gross evaluation is shown in Table 1 .

\section{Histopathological examination}

Following the macroscopic evaluation, an overdose of the anesthetic agent was administered to all the rats as euthanasia. The whole sciatic nerve and surrounding tissue, including the repaired site and NN, were removed en bloc. The neural tissue specimens were kept in a $10 \%$ formalin solution for 24 hours and were then prepared with ethanol and xylene before being embedded in paraffin. Slices of 3-micrometer thickness were cut from the paraffin

\section{Tab. 1. Numerical Grading Scheme for the Gross Evaluation.}

\begin{tabular}{lcl}
\hline Tissue & Grade & Definition \\
\hline \multirow{2}{*}{ Skin and muscle } & 1 & Skin or muscle fascia entirely closed \\
fascia & 2 & Skin or muscle fascia partially open \\
& 3 & Skin or muscle fascia completely open \\
\hline \multirow{3}{*}{ Nerve adherence } & 1 & No dissection or mild blunt dissection \\
& 2 & Some vigorous blunt dissection required \\
& 3 & Sharp dissection required \\
\hline
\end{tabular}


Tab. 2. Scale for the Histomorphological Organization of the Regenerating Nerve.

\begin{tabular}{cl}
\hline Grade & Definition \\
\hline 1 & $\begin{array}{l}\text { Failure, no continuity of the axons from the proximal } \\
\text { to distal ends }\end{array}$ \\
\hline 2 & $\begin{array}{l}\text { Poor organization (interlacing or whirling appearance } \\
\text { of the nerve fibers) }\end{array}$ \\
\hline 3 & $\begin{array}{l}\text { Fair organization (focal whirling appearance, focal parallel, } \\
\text { alignment) }\end{array}$ \\
\hline 4 & $\begin{array}{l}\text { Good organization, approaching normal (mostly parallel, } \\
\text { without a whirling or wavy appearance) }\end{array}$ \\
\hline 5 & $\begin{array}{l}\text { Excellent organization of the repair site, indistinguishable } \\
\text { from normal }\end{array}$ \\
\hline
\end{tabular}

blocks and were stained with hematoxylin-eosin. All examinations were applied by two experienced histopathologists independently of each other using the same light microscope (Zeiss, Oberkochen, Germany).

\section{Counts of perineural fibroblasts and inflammatory cells}

The analysis was made of the cellular components to determine the number of fibroblasts and inflammatory cells around the repaired area of the damaged nerve. The fibroblasts and inflammatory cells were counted from 4 different quadrants of the perineural scar tissue around the repair site at $x 400$ magnification. The numbers of fibroblasts and inflammatory cells were counted according to the principles described by Park et al (16).

\section{Scar tissue formation index}

Quantitative analysis of the scar tissue was applied by measurement with a microscope of the thickness of the thickest area of scar tissue. This value was then divided by the thickness of the nerves in the same area to provide a standardized ratio. The value obtained represented the scar tissue formation index according to the principles described by Albayrak et al (17).

\section{Histomorphological organization of axons}

Using the previously-described scale by Brown et al, longitudinal organization and morphology of the axon at the nerve repair site was evaluated (18). The scale is scored from 1 to 5 as 1 $=$ failure, no continuity of the axons from the proximal to distal ends, 2 = poor organization of the repair site, 3 = fair organization of the repair site, $4=$ good organization of the repair site, approaching normal and $5=$ excellent organization of the repair site, indistinguishable from normal. Table 2 displays the scale for the histomorphological organization of the regenerating nerve.

\section{Number of axons}

From each sample, 20 histopathological sections of 3-micrometer thickness were cut with an ultramicrotome. These were stained with thionine and photographs were taken under light microscopy (Olympus BX51, Olympus Corp., Tokyo, Japan). The number of axons was counted with a digital counter in 6 zones (1 from the central zone, 5 from peripheral zones) under x10 magnification according to the principles described by Kücük et al (ThermoScientific, Finland) and a computer program (Skanlt for Multiscan FC2.5.1). The results were expressed in picogram per gram tissue (19).

\section{Statistical analysis}

Data were analyzed using the IBM Statistical Package for Social Sciences version 20 (SPSS Inc., Chicago, IL, USA). Kruskal-Wallis test was used to compare more than two independent groups. If a significant difference was detected between groups, MannWhitney U test was employed. Categorical variables were analyzed using Pearson Chi-Square test. Quantitative variables were expressed as a median and interquartile range. The level of confidence was $95 \%$ and p-value less than 0.05 .

\section{Results}

Pearson Chi-Square test revealed that there was a significant difference between the degrees of perineural adhesion between groups ( $p=0.026)$. Groups I, III, V and VII exhibited a more severe degree of perineural adhesion compared to Groups II, IV, VI, and VIII. In other words, both crush and transection nerve injury groups receiving EPO either locally or intraperitoneally had less a prominent degree of perineural adhesion (Figs 3a, b, c). On the other hand, a histomorphological organization of axons was similar between eight experimental groups $(p=0.126)$.

Kruskal-Wallis test yielded that there were significant differences between groups with respect to scar tissue formation index, inflammatory cell count, perineural fibroblast count, the number of axons, tissue levels of IL-1 $\beta$, IL-6, and IL-10.

Scar tissue formation index was remarkably lower in Groups II, IV, VI and VIII compared to Groups I, III, V and VII ( $\mathrm{p}<$ 0.001 ). Inflammatory cell and perineural fibroblast counts were significantly higher in Groups I, III, V and VII $(p<0.001$ for both
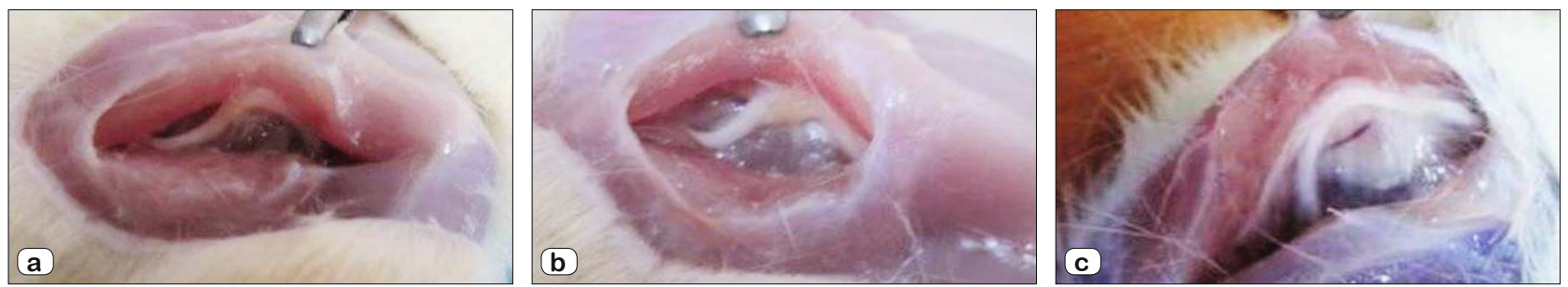

Fig. 3 a, b, c. Mild perineural adhesion is observed in groups receiving EPO (a, b), whereas severe perineural adhesion is evident in control groups that had isotonic saline injections (c). 

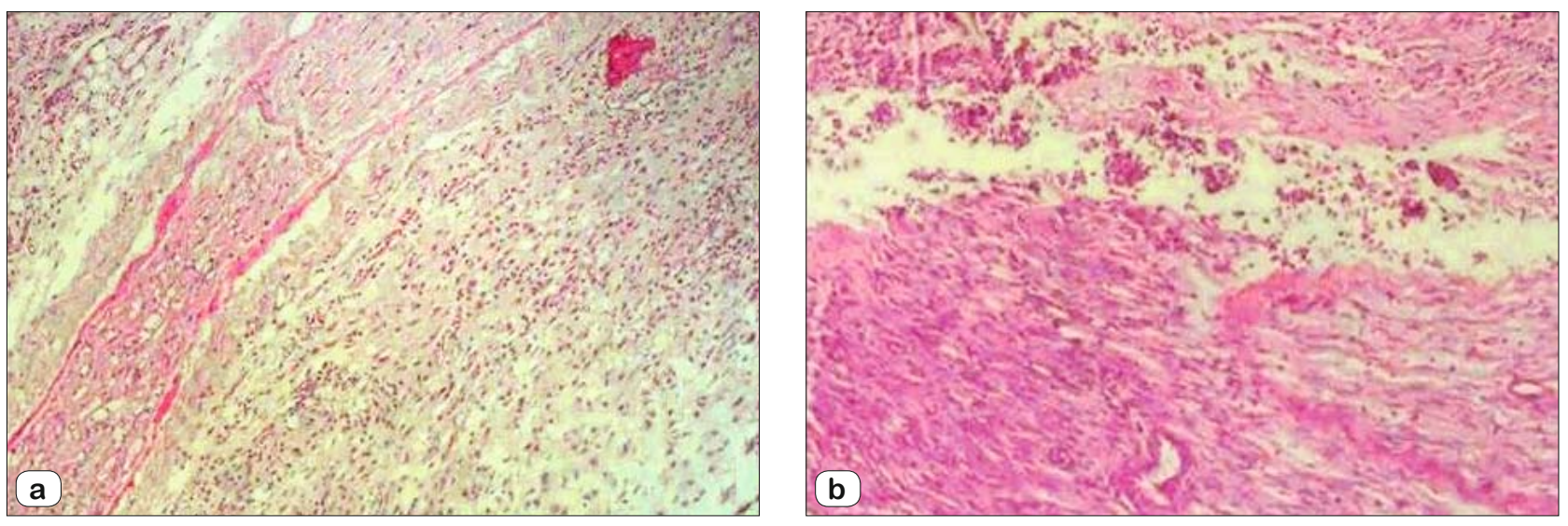

Fig. 4 a, b. Perineural scar formation, fibroblast and inflammatory cell infiltration are obvious after crush injury (a). These effects are less prominent in groups receiving EPO (b).

parameters) (Figs 4a, b). The number of axons were significantly increased in Groups II, IV, VI and VIII $(\mathrm{p}<0.001)$

Tissue levels of pro-inflammatory cytokines, IL-1 $\beta$ and IL-6, were significantly lower in Groups II, IV, VI and VIII $(\mathrm{p}<0.001)$, whereas the level of anti-inflammatory cytokine, IL-10, was higher in these groups $(\mathrm{p}<0.001)$.

A comparative overview of histopathological parameters under investigation and tissue levels of interleukins is presented in Table 3.

\section{Discussion}

Peripheral nerves may often be vulnerable to various types of injury such as crushing, compression, stretching, avulsion, and division. Attributed to the degeneration of motor neurons, the lack of an appropriate environment fort he survival of Schwann cells and the limited capacity of the nerves for regeneration, peripheral nerve injuries are accompanied with substantial functional deficits particularly in case axons are unable to reestablish continuity with the distal nevre (13). Iatrogenic peripheral nerve trauma constitutes a remarkable source of morbidity and remains a challenge for clinicians (13). Nerve damage during surgery causes significant complications. this complication significantly affects the surgical success and comfort of the patient. Effective study was not performed in iatrogenic nerve injury treatment.

The purpose of the present study was to investigate the histopathological and inflammatory changes attributed to the topical and systemic use of EPO after transection and crush type peripheral nerve injuries. Our results demonstrated that both local and systemic administrations of EPO were associated with attenuation of the inflammatory response, reduction of tissue levels of proinflammatory cytokines IL-1 $\beta$, IL-6 and increase in levels of antiinflammatory cytokine IL-10. These beneficial impacts of EPO did not vary with respect to the route of administration (locally or intraperitoneally) or according to the type of injury (crush or transection). Therefore, we suggest that EPO may have a promising potential to be used as a therapeutic alternative in crush or transection types of peripheral nerve injuries. The significant difference in axon numbers between groups receiving saline and EPO may be due to enhancement of axonal regrowth and promotion of functional recovery after peripheral injury in rat models $(20,21)$. However, the exact mechanism of action for EPO in repair process after peripheral nerve injury remains to be elucidated in further trials.

The neuroprotective role of EPO, an endogenous hormone and FDA-approved drug for the treatment of anemia, has been welldocumented by many trials focusing on a wide range of neurological problems. Similar to the present study, multiple animal trials have been conducted for peripheral nerve injury (21-28). Recently, beneficial effects of EPo after peripheral nerve injury has been demonstrated in humans as well (29). However, the vast majority of these trials have investigated either a single type or injury or EPO has been employed using either local or systemic route. Therefore, this study is unique and original since it assesses different types of nerve injuries and various routes of administration together.

Erythropoietin exerts its protective effects by decreasing neuroinflammation and neuronal death (30). It is supposed to induce neurorepair by enhancement of neurogenesis, angiogenesis, and promotion of synaptic plasticity (30). Our findings are consistent with this data since we observed an apparent difference between axon numbers in groups receiving saline and EPO.

Protective role of EPO using reversal of vascular spasm is dependent on the inhibition of nitric oxide production (31). It may also reduce the inflammatory infiltrate and display neuroprotective effects from glutamate toxicity by activation of calcium channels, production of antioxidant enzymes in neurons and neoangiogenesis, which improves blood flow and tissue oxygenation across an ischemic area (31). Our results yielded that EPO may decrease levels of proinflammatory cytokines, Il-1 $\beta$ and IL-6; and increase the level of IL-10, an anti-inflammatory cytokine. Erythropoietin is a safe and well-tolerated agent which can be administered systemically. Since the protective action of EPO lasts only approximately three days, duration of administration should be longer for the treatment of neurological diseases. On the other hand, side effects may be seen due to long-term administration of EPO. The long-term consequences of lengthy EPO treatment may include polycythemia (production of hyperreactive platelets), 
Tab. 3. Comparison of experimental groups with respect to histopathological alterations.

\begin{tabular}{|c|c|c|c|c|}
\hline Variable & Group & $\mathrm{n}$ & Median-IQR & $\mathrm{p}$ \\
\hline \multirow{8}{*}{$\begin{array}{l}\text { Scar tissue } \\
\text { formation index }\end{array}$} & I & 8 & $0.19-0.020$ & \multirow{8}{*}{$<0.001 *$} \\
\hline & II & 8 & $0.07-0.020$ & \\
\hline & III & 8 & $0.19-0.015$ & \\
\hline & IV & 8 & $0.07-0.010$ & \\
\hline & $\mathrm{V}$ & 8 & $0.20-0.018$ & \\
\hline & VI & 8 & $0.07-0.020$ & \\
\hline & VII & 8 & $0.19-0.018$ & \\
\hline & VIII & 8 & $0.07-0.020$ & \\
\hline \multirow{8}{*}{$\begin{array}{l}\text { Inflammatory cell } \\
\text { count }\end{array}$} & I & 8 & $60-2.75$ & \multirow{8}{*}{$<0.001 *$} \\
\hline & II & 8 & $18-1.75$ & \\
\hline & III & 8 & $61-1.75$ & \\
\hline & IV & 8 & $24-3.25$ & \\
\hline & V & 8 & $58-3.50$ & \\
\hline & VI & 8 & $21-2.50$ & \\
\hline & VII & 8 & $57-1.75$ & \\
\hline & VIII & 8 & $22-1.75$ & \\
\hline \multirow{8}{*}{$\begin{array}{l}\text { Perineural fibroblast } \\
\text { count }\end{array}$} & I & 8 & $144.5-3.25$ & \multirow{8}{*}{$<0.001^{*}$} \\
\hline & II & 8 & $66.0-2.75$ & \\
\hline & III & 8 & $145.0-2.75$ & \\
\hline & IV & 8 & $72.0-1.75$ & \\
\hline & V & 8 & $139.5-3.75$ & \\
\hline & VI & 8 & $62.0-1.75$ & \\
\hline & VII & 8 & $140.0-2.75$ & \\
\hline & VIII & 8 & $69.5-2.50$ & \\
\hline \multirow{8}{*}{ Number of axons } & I & 8 & $878-6.0$ & \multirow{8}{*}{$<0.001^{*}$} \\
\hline & II & 8 & $1598-7.5$ & \\
\hline & III & 8 & $874-3.0$ & \\
\hline & IV & 8 & $1487-9.0$ & \\
\hline & $\mathrm{V}$ & 8 & $834-3.0$ & \\
\hline & VI & 8 & $1560-4.0$ & \\
\hline & VII & 8 & $828-5.5$ & \\
\hline & VIII & 8 & $1442-5.5$ & \\
\hline \multirow{8}{*}{ IL-1 $\beta$} & I & 8 & $194-3.5$ & \multirow{8}{*}{$<0.001^{*}$} \\
\hline & II & 8 & $132-3.5$ & \\
\hline & III & 8 & $186-5.5$ & \\
\hline & IV & 8 & $118-3.5$ & \\
\hline & $\mathrm{V}$ & 8 & $188-3.5$ & \\
\hline & VI & 8 & $139-3.5$ & \\
\hline & VII & 8 & $190-4.0$ & \\
\hline & VIII & 8 & $114-3.5$ & \\
\hline \multirow{8}{*}{ IL-6 } & I & 8 & $1872-12.0$ & \multirow{8}{*}{$<0.001 *$} \\
\hline & II & 8 & $779-7.0$ & \\
\hline & III & 8 & $1842-8.0$ & \\
\hline & IV & 8 & $764-5.3$ & \\
\hline & V & 8 & $1855-5.0$ & \\
\hline & VI & 8 & $781-3.5$ & \\
\hline & VII & 8 & $1865-8.5$ & \\
\hline & VIII & 8 & $744-3.8$ & \\
\hline \multirow{8}{*}{ IL-10 } & I & 8 & $87.5-3.75$ & \multirow{8}{*}{$<0.001 *$} \\
\hline & II & 8 & $138-3.50$ & \\
\hline & III & 8 & $92.0-3.25$ & \\
\hline & IV & 8 & $146.0-2.75$ & \\
\hline & $\mathrm{V}$ & 8 & $90.0-3.25$ & \\
\hline & VI & 8 & $140.0-4.00$ & \\
\hline & VII & 8 & $90.0-3.00$ & \\
\hline & VIII & 8 & $150.0-3.25$ & \\
\hline
\end{tabular}

which can predispose the patient to thrombosis, especially in the setting of injury (31).

A strength of the present study is a simultaneous assessment of local or systemic administration of EPO on two types of injury (transection or crush) with a focus on both histopathological and biochemical aspects. Therefore, we hope that our results may provide multi-dimensional insights on the beneficial and protective effects of EPO after crush and transection types of peripheral nerve injuries. Thereby, support for the development of evidencebased approaches on the route of application for different indications can be possible.

In accordance with our data, substantial information exists on the neuroprotective and neuroregenerative capacity of EPO in the treatment of peripheral nerve injury. Campana et al have shown that neuropathic pain symptoms were relieved in rats after chronic crush injuries (22). Furthermore, administration of EPO was accompanied with diminution of the outcomes of nerve injury such as axonal degeneration, increased TNF- $\alpha$ levels which lead to pain and Schwann cell apoptosis (22). Elfar et al demonstrated that EPO improved the sciatic function index of mice after calibrated crush injury (32). Additionally, this effect was observed with administration before injury, immediately after the injury, and after one week, suggesting that timing is not critical (32). Li et al reported similar findings of increased EPO production as well as receptor upregulation on Schwann cells in the setting of chronic sciatic nerve injury in rats (25). Rotter et al demonstrated the regenerative benefits of EPO on motor strength, pain behavior, and nerve conduction velocity in rats exposed to crush injury of the sciatic nevre (26). Grasso et al found that EPO improved recovery time in rats with sciatic nerve crush injuries (23). In conjunction with the report by Inoue et al, our histopathological findings support that EPO may facilitate the recovery by induction of Schwann cell migration to sites of nerve injury (24). Our data supports that both local and intraperitoneal routes are effective to achieve the neuroprotective effects of EPO. In parallel with the results of Yin et al we noted that EPO decreased scar formation and alleviated the inflammatory response that may hinder regeneration after crush or transection type peripheral nerve injuries (21). Lykissas has shown improved motor function in rats treated with EPO after sharp transection and end-to-side neurorrhaphy (28).

In spite of the promising results of our study, it must be remembered that EPO has certain side effects such as involvement in regulation of tumor angiogenesis, hypertension, polycythemia, myocardial infarction, stroke and seizures, thromboembolism and immunogenicity (5).

As with all studies, this trial possesses some weaknesses. First, we have not evaluated the sensory or motor function. Inflammatory indicators are vulnerable to be influenced by various metabolic, environmental and genetic conditions at histopathological and biochemical levels. Moreover, the study is subject to the usual limitations associated with experimental studies. In addition, the dose of EPO has not been standardized and no valid conclusions regarding ideal dosing can be made.

\section{Conclusion}

To conclude, iatrogenic nerve injury has remained an area with few therapeutic options. Our results indicated that local and systemic applications of EPO might have a promising potential 
therapeutic agent for crush or transection type of peripheral nerve injuries. Dose, route of administration and indications should be elucidated in further prospective, randomized, controlled trials.

\section{References}

1. Burnett MG, Zager EL. Pathophysiology of peripheral nerve injury: a brief review. Neurosurg Focus 2004; 16: E1.

2. Rishal I, Fainzilber M. Retrograde signaling in axonal regeneration. Exp Neurol 2010; 223: 5-10.

3. Bridge PM, Ball DJ, Mackinnon SE, Nakao Y, Brandt K. Nerve crush injuries a model for axonotmesis. Exp Neurol. 1994; 127: 284-290.

4. Hobbenaghi R, Javanbakht J, Hosseini E, Mohammadi S, Rajabian M, Moayeri $\mathbf{P}$ et al. Neuropathological and neuroprotective features of vitamin B12 on the dorsal spinal ganglion of rats after the experimental crush of sciatic nerve: an experimental study. Diagn Pathol 2013; 8: 123 .

5. Sanchis-Gomar F, Perez-Quilis C, Lippi G. Erythropoietin receptor (EpoR) agonism is used to treat a wide range of disease. Mol Med 2013; 19: 62-64.

6. Carraway MS, Suliman HB, Jones WS, Chen CW, Babiker A, Piantadosi CA. Erythropoietin activates mitochondrial biogenesis and couples red cell mass to mitochondrial mass in the heart. Circ Res 2013; 106: $1722-1730$

7. Lundby C, Olsen NV. Effects of recombinant human erythropoietin in normal humans. J Physiol 2011; 589: 1265-1271.

8. Campana WM, Myers RR. Erythropoietin and erythropoietin receptors in the peripheral nervous system: changes after nerve injury. FASEB J 2001; 15: 1804-1806.

9. Bianchi R, Buyukakilli B, Brines M, Savino C, Cavaletti G, Oggioni $\mathbf{N}$ et al Erythropoietin both protects from and reverses experimental diabetic neuropathy. Proc Natl Acad Sci USA 2004; 101: 823-828.

10. Campana WM, Myers RR. Exogenous erythropoietin protects against dorsal root ganglion apoptosis and pain following peripheral nerve injury. Eur J Neurosci 2003; 18: 1497-1506.

11. Keswani SC, Buldanlioglu U, Fischer A, Reed N, Polley M, Liang H et al. A novel endogenous erythropoietin mediated pathway prevents axonal degeneration. Ann Neurol 2004; 56: 815-826.

12. Lykissas MG, Korompilias AV, Vekris MD, Mitsionis GI, Sakellariou E, Beris AE. The role of erythropoietin in central and peripheral nerve injury, review. Clin Neurol Neurosurg 2007; 109: 639-644.

13. Wang W, Li D, Li Q, Wang L, Bai G, Yang T et al. Erythropoietin promotes peripheral nerve regeneration in rats by upregulating expression of insulin-like growth factor-1. Arch Med Sci 2015; 11: 433-437.

14. Yu H, Liu J, Ma J, Xiang L. Local delivery of controlled released nerve growth factor promotes sciatic nerve regeneration after crush injury. Neurosci Lett 2014; 566: 177-181.

15. Petersen J, Russell L, Andrus K, MacKinnon M, Silver J, Kliot M. Reduction of extraneural scarring by ADCON-T/N aft er surgical intervention. Neurosurgery. 1996; 38: 976-983.

16. Park SJ, Lee HJ, Han CS, Chung DW, Kim GY. Effect of hyaluronic acid-carboxymethylcellulose solution on perineural scar formation after sciatic nerve repair in rats. Clin Orthop Surg 2011; 3: 315-324.
17. Albayrak BS, Ismailoglu O, Ilbay K, Yaka U, Tanriover G, Gorgulu A et al. Doxorubicin for prevention of epineurial fibrosis in a rat sciatic nerve model: outcome based on gross postsurgical, histopathological, and ultrastructural findings. J Neurosurg Spine 2010; 12: 327-333.

18. Brown RE, Erdmann D, Lyons SF, Suchy H. The use of cultured Schwann cells in nerve repair in a rabbit hind-limb model. J Reconstr Microsurg 1996; 12: 149-152.

19. Kü̧̈ük L, Günay H, Erbaş O, Küçük Ü, Atamaz F, Coşkunol E. Effects of platelet-rich plasma on nerve regeneration in a rat model Acta Orthop Traumatol Turc 2014; 48: 449-454.

20. Zhang W, Sun B, Yu Z, An J, Liu Q, Ren T. High dose erythropoietin promotes functional recovery of rats following facial nerve crush. J Clin Neurosci 2009; 16: 554-556.

21. Yin ZS, Zhang H, Bo W, Gao W. Erythropoietin promotes functional recovery and enhances nerve regeneration after peripheral nerve injury in rats. Am J Neuroradiol 2010; 31: 509-515.

22. Campana WM, Li X, Shubayev VI, Angert M, Cai K, Myers RR. Erythropoietin reduces schwann cell TNF-a, Wallerian degeneration and pain-related behaviors after peripheral nerve injury. Eur J Neurosci 2006; 23: 617-626.

23. Grasso G, Meli F, Fodale V, Calapai G, Buemi M, Iacopino D. Neuroprotective potential of erythropoietin and darbepoetin alfa in experimental model of sciatic nerve injury. J Neurosurg Spine 2007; 7: 645-651.

24. Inoue G, Gaultier A, Li X, Mantuano E, Richardson G, Takahashi $\mathrm{K}$ et al. Erythropoietin promotes schwann cell migration and assembly of the provisional extracellular matrix by recruiting Betal integrin to the cell surface. Glia 2010; 58: 399-409.

25. Li X, Gonias SL, Campana WM. Schwann cells express erythropoietin receptor and represent a major target for epo in peripheral nerve injury. Glia 2005; 51: 254-265.

26. Rotter R, Kuhn C, Stratos I, Beck M, Mittlemeier T, Vollmar B. Erythropoietin enhances the regeneration of traumatized tissue after combined muscle-nerve injury. J Trauma Acute Care Surg 2012; 72: 1567-1575.

27. Toth C, Martinez JA, Liu WQ, Diggle J, Guo GF, Ramji N et al. Local erythropoietin signaling enhances regeneration in peripheral axons. Neuroscience 2008; 154: 767-783.

28. Lykissas MG, Sakellariou E, Vekris MD, Kontogeorgakos VA, Batistatou AK, Mitsionis GI et al. Axonal regeneration stimulated by erythropoietin: an experimental study in rats. J Neurosci Methods 2007; 164: 107-115.

29. Bernstein DT, Weiner BK, Tasciotti E, Mathis KB. Does the combination of erythropoietin and tapered oral corticosteroids improve recovery following iatrogenic nerve injury? Injury 2016; 47: 1819-1823.

30. Cervellini I, Annenkov A, Brenton T, Chernajovsky Y, Ghezzi P, Mengozzi M. Erythropoietin (EPO) increases myelin gene expression in CG4 oligodendrocyte cells through the classical EPO receptor. Mol Med 2013; 19: 223-229.

31. Grasso G, Sfacteria A, Erbayraktar S, Passalacqua M, Meli F, Gokmen $\mathbf{N}$ et al. Amelioration of spinal cord compressive injury by pharmacological preconditioning with erythropoietin and a nonerythropoietic erythropoietin derivative. J Neurosurg Spine 2006; 4: 310-318.

32. Elfar JC, Jacobson JA, Puzas JE, Rosier RN, Zuscik MJ. Erythropoietin accelerates functional recovery after peripheral nerve injury. JBJS 2008; 90: 1644-1653. 